\title{
French validation of the Barcelona music reward questionnaire
}

Joe Saliba, Urbano Lorenzo-Seva, Josep Marco-Pallares, Barbara Tillman, Anthony Zeitouni, Alexandre Lehmann

Background. The Barcelona Music Reward Questionnaire (BMRQ) questionnaire investigates the main facets of music experience that could explain the variance observed in how people experience reward associated with music. Currently, only English and Spanish versions of this questionnaire are available. The objective of this study is to validate a French version of the BMRQ. Methods. The original BMRQ was translated and adapted into an international French version. The questionnaire was then administered through an online survey aimed at adults aged over 18 years, fluent in French. Statistical analyses were performed and compared to the original English and Spanish version for validation purposes. Results. A total of 1027 participants completed the questionnaire. Most responses were obtained from France (89.4\%). Analyses revealed that congruence values between the rotated loading matrix and the ideal loading matrix ranged between 0.88 and 0.96 . Factor reliabilities of subscales (i.e., Musical Seeking, Emotion Evocation, Mood Regulation, Social Reward and Sensory-Motor) also ranged between 0.88 and 0.96. In addition, reliability of the overall factor score (i.e., Music reward) was 0.91 . Finally, the internal consistency of the overall scale was 0.85 . The factorial structure obtained in the French translation was similar to that of the original Spanish and English samples.

Conclusion. The French version of the BMRQ appears valid and reliable. Potential applications of the BMRQ include its use as a valuable tool in music reward and emotion research, whether in healthy individuals or in patients suffering from a wide variety of cognitive, neurologic and auditory disorders. 


\section{French Validation of the Barcelona Music Reward Questionnaire}

2

3 Joe Saliba, $\mathrm{MD}^{1}$, Urbano Lorenzo-Seva, $\mathrm{PhD}^{2}$, Josep Marco-Pallares, $\mathrm{PhD}^{3}$, Barbara Tillmann, $4 \mathrm{PhD}^{4}$, Anthony Zeitouni, MD, FRSCS${ }^{1}$, Alexandre Lehmann, $\mathrm{PhD}^{1,5}$

5

6 Institutions:

7
1. Department of Otolaryngology-Head \& Neck Surgery, McGill University, Montreal, Canada.

2. Research Center for Behavior Assessment, Universitat Rovira i Virgili, Tarragona, Spain

3. Department of Basic Psychology, University of Barcelona, Barcelona, Spain.

4. Lyon Neurosciences Research Center, University Lyon-1, Lyon, France

5. Center for Research on Brain, Language and Music; International Laboratory for Brain, Music and Sound Research, QC, Canada

$\underline{\text { Abstract }}$

Background. The Barcelona Music Reward Questionnaire (BMRQ) questionnaire investigates the main facets of music experience that could explain the variance observed in how people experience reward associated with music. Currently, only English and Spanish versions of this questionnaire are available. The objective of this study is to validate a French version of the BMRQ.

Methods. The original BMRQ was translated and adapted into an international French version. The questionnaire was then administered through an online survey aimed at adults aged over 18 years, fluent in French. Statistical analyses were performed and compared to the original English and Spanish version for validation purposes. 
26 Results. A total of 1027 participants completed the questionnaire. Most responses were obtained from

27 France (89.4\%). Analyses revealed that congruence values between the rotated loading matrix and the

28 ideal loading matrix ranged between 0.88 and 0.96 . Factor reliabilities of subscales (i.e., Musical Seeking,

29 Emotion Evocation, Mood Regulation, Social Reward and Sensory-Motor) also ranged between 0.88 and 30 0.96. In addition, reliability of the overall factor score (i.e., Music reward) was 0.91 . Finally, the internal

31 consistency of the overall scale was 0.85 . The factorial structure obtained in the French translation was

32 similar to that of the original Spanish and English samples.

33 Conclusion. The French version of the BMRQ appears valid and reliable. Potential applications of the

34 BMRQ include its use as a valuable tool in music reward and emotion research, whether in healthy

35 individuals or in patients suffering from a wide variety of cognitive, neurologic and auditory disorders.

37 Corresponding author:

39 Dr Alexandre Lehmann, PhD

40 Faculty of Medicine, Department of Otolaryngology-Head \& Neck Surgery

41 McGill University Health Center, Suite DO5.5713

421001 Decarie Boulevard

43 H4A3J1, Montréal, Qc 


\section{Introduction}

55

56 The rewarding effects of music are highly dependent on cultural and personal preferences. As a

57 result, large differences in the way individuals experience musical pleasure are observed (Blood and Zatorre, 2001; Chanda and Levitin, 2013). While music can induce positive effects on mood and affect in some individuals (Juslin \& Västfjäll, 2008), others seek the social bonding capacity of music (Cross, 2001). Conversely, certain individuals cannot experience any pleasure from widely different stimuli, including music - a disorder termed anhedonia (Assogna et al., 2011). Traditionally, it has been hard to assess the sources of this inter-individual variability in musicinduced reward. Previous groups have developed questionnaires - such as the BIS/BAS scales

64 (Carver and White, 1994) or the Sensitivity to Reward/Sensitivity to Punishment Questionnaire 65 (Torrubia, 2001) - that assess individual differences to overall sensitivity to reward experiences

66 (Carver \& White, 1994; Torrubia et al., 2001). However, music is considered as a higher-order

67 pleasure and as such, might involve different processing mechanisms than basic rewards (Menon $68 \&$ Levitin, 2005). In addition, previous studies have supported a dissociation of music rewarding 69 experience from other rewarding experiences related to other types of primary and secondary 70 reinforcements, such as food, sex and money among others (Mas-Herrero et al., 2014).

71 In light of these findings, the Barcelona Music Reward Questionnaire (BMRQ) was developed

72 by Mas-Herrero et al. (2013). This questionnaire is specifically geared towards assessing 73 sensitivity to music reward and was a welcome addition to a limited choice of behavioral tools

74 suitable for music reward studies. The BMRQ can serve as a valuable research tool in 75 psychophysical studies addressing music reward in healthy individuals, hearing-impaired 76 individuals or individuals affected with other conditions or pathologies. To date, only Spanish 
77 and English versions of this questionnaire are available in the literature, limiting its application.

78 With over 200 million speakers worldwide, French is one of the most common languages in the

79 world. In countries with several official languages including French such as Canada, Morocco

80 and Senegal, it is all the more important for research tools to be available in all official languages

81 to adequately test the population. In that context, a French version of the BMRQ was required to

82 meet the needs of the numerous researchers in the French-speaking areas of the world. In this

83 paper, we sought to translate the Barcelona Music Reward Questionnaire into an international

84 French and to assess its construct validity and reliability.

85

86 Materials and Methods

87

The Barcelona Music Reward Questionnaire

89

90 The BMRQ examines five main facets that characterize musical reward experience in

91 individuals: musical seeking, emotion evocation, mood regulation, social reward and sensory-

92 motor. Musical seeking refers to the way individuals pursue music-related activities (attending

93 concerts, playing an instrument) or seek additional information about music they listen to

94 (performers, composers). The emotion evocation aspect of music reward is related to the

95 emotional impact of music on individuals. In contrast, the ability of listeners to use music to

96 modulate their emotions (to relieve stress, to release emotions, to comfort) is referred to as mood

97 regulation. The social reward facet examines the social bonding effect of music on individuals.

98 Lastly, the sensory-motor facet addresses the capacity of music to intuitively induce body

99 movements synchronized to a rhythm's beat in certain individuals (head nodding, even dancing). 
100 The questionnaire contains 20 statements equally divided among these five facets. Participants

101 indicate the level of agreement with each statement by using a five-point scale ranging from

102 "fully disagree", (1) to "fully agree", (5). The contribution of each facet to the overall music

103 reward experience is quantified by a numerical value obtained upon completion of the survey. A

104 score for global sensitivity to music reward is also provided, which was obtained as the weighted

105 sum of participants' scores (i.e factor score). The mean value of each factor is 50, and the

106 standard deviation is 10 . Standard values are therefore located between 40 and 60 . Punctuations

107 below 40 indicate low values in this particular facet, whereas values above 60 indicate high

108 values (the same applies to the global sensitivity to music reward) (Mas-Herrero et al., 2013).

109

110 The BMRQ was created in three steps (Mas-Herrero et al., 2013). The first consisted in

111 developing of a short psychometric instrument in Spanish that included various facets of music

112 and reward experiences. This initial instrument included 112 items addressing a variety of

113 activities and situations associated with music reward and pleasure experiences, and was

114 administered to 804 Spanish participants. From the initial pool of 112 items, only 20 were

115 retained for the final version, equally divided among five facets of music reward (music seeking

116 activities, mood regulation, emotion evocation, sensory-motor behavior and social reward).

117 Selection was based on loading values and content and adequacy of the items. The second step

118 involved exploratory and confirmatory factorial analysis of the Spanish BMRQ. The

119 questionnaire with the selected 20 items was administered to a new sample of 605 students in an

120 effort to replicate the previous findings. Analyses revealed a reliable factorial structure for the

121 Spanish BMRQ and an acceptable fit for the hypothesized five facets of music reward. The final

122 step in the development of the BMRQ was its translation and adaptation into English. The 
123 translated version was completed by 252 English-speaking participants, and confirmatory

124 factorial analysis was performed to verify the replicability of the factor structure obtained in the

125 Spanish version. The original BMRQ has been shown to be valid with acceptable reliability

126 estimates of factor scores (i.e., a reliability value of 0.93 for the overall scale, and values

127 between 0.73 and 0.93 for the five subscales). The aim of the present study was to replicate this 128 validation for a French adaptation of the BMRQ.

130 Questionnaire translation

131

132 The French adaptation of the BMRQ was obtained by forward and backward translation. Each

133 item in the original English version of the questionnaire was independently translated by two 134 groups two of bilingual (French and English) researchers - in Montreal, Canada and in Lyon,

135 France - whose first language was French. Both groups also had knowledge of the subject

136 matter. The groups were purposely chosen in different geographic areas in order to account for

137 the regional differences in spoken French and hence create an internationally comprehensible

138 French translation. The Spanish questionnaire was used as a reference for disambiguating some

139 wordings. The emphasis was placed on the translation of meaning rather than a literal one. A

140 consensus between the two translator groups was obtained to produce the final French version of

141 the questionnaire. Finally, a third bilingual researcher (French and Spanish) conducted a back

142 translation into Spanish. This researcher was not involved in the initial translation process. This

143 last step ensured the meaning of the adapted French version was concordant with the meaning of

144 the original Spanish questionnaire. The comparison between the source items and the French

145 translation is shown in Appendix 1. The content of the French translation of the BMRQ is 
146 reproduced in Appendix 2, along with the complete set of instructions, thus allowing readers for

147 a direct use of this questionnaire tool.

148

149 Data collection and participants

150

151 The questionnaire was administered via an Internet platform (LimeSurvey, McGill University

152 servers) to any participant aged over 18 years and fluent in French. A written electronic consent

153 was obtained for each participant. The survey was made publicly accessible from November

1542014 to April 2015 and distributed electronically through various academic and healthcare

155 institutions mainly in Europe, North America and Africa, but also in other areas of the world. In

156 order to avoid sampling bias effect, the music focus of the study was not explicitly stated in the

157 test instructions when administered to participants. Prior to completing the survey, participants

158 were also asked to fill out a general demographic and linguistic background questionnaire. This

159 study was approved by the McGill University’s Faculty of Medicine Institutional Review Board 160 (\#A11-E88-14B).

161

162 Evaluation of the Psychometric Properties of the Translated Version

163

164 In order to assess the structure validity of the test, we used an approach similar to that employed

165 by Mas-Herrero and colleagues in the development of the original questionnaire, as described

166 above (Mas-Herrero et al., 2013). An exploratory factor analysis was carried out using

167 MATLAB, and, for scale analyses, SPSS 22 was used. The polychoric correlation matrix was

168 computed for the 20 items of the translated questionnaire. To control the variance due to this 
169 response style factor, we applied the procedure proposed by Lorenzo-Seva and Rodriguez-

170 Fornells (2006) developed for the specific case of non-perfectly balanced scales (see Lorenzo-

171 Seva \& Ferrando, 2009). As five content factors were expected, we retained this number of

172 factors using Minimum Rank Factor Analysis (MRFA, Ten Berge \& Kiers, 1991). Observed

173 variables in MRFA consist of common parts and unique parts, each satisfying certain

174 requirements: the covariance matrices for common and unique parts are positive semidefinite,

175 and the unique-parts covariance matrix is diagonal. An oblique semi-specified Procrustean

176 rotation (Browne, 1972) was performed in order to establish the loading factors associated with

177 each of the five content factors. For the purposes of this analysis, the specified values were the

178 loadings on each item that we expected to be zero. The procedure reported by Ten Berge et al.

179 (Ten Berge et al., 1999) was then employed to calculate factor scores. The mean and

180 standardized deviation of items, and the factor weights required to compute these factor scores

181 are available for the use of researchers (in the supplementary materials and on the online test

182 page).

183

184 We computed the reliability estimates for the five scales and the total scale on the basis of the factor 185 scores based on the factor scores reliability (for example, see Mellenbergh, 1994; formula 22 on 186 page 231). To assess internal consistency, we computed Cronbach's alpha for the overall scale.

\section{$188 \underline{\text { Results }}$}

189

190 A total of 1027 participants voluntarily completed the entire translated questionnaire (Mean age:

19122.3 (SD 7.8) years, females: 64.7\%). While participants were mostly from France (89.4\%) and 
192 Canada (5.1\%), 4\% of our sample was obtained from 25 other countries such as Cameroun,

193 Senegal and Egypt. Table 1 resumes the demographic statistics of the sample. The majority of

194 our respondents were non-musicians (77.5\%). While the questionnaire was primarily advertised

195 in academic institutions, approximately a quarter of our participants did not complete a

196 university degree. Overall, our French sample was similar to the Spanish and English samples in

197 terms of age, gender and music training.

198

199 Table 2 shows the means and standard deviations of items of the French and the Spanish version 200 of the test. As can be observed, the differences observed between the mean items in both cultures 201 were not significant.

202

203 Once the polychoric correlation matrix was available, the observed Kaiser-Meyer-Olkin (KMO, 204 Kaiser, 1970) index was computed: the 0.855 value obtained suggested that the correlation 205 matrix was well suited for factor analysis (see Kaiser \& Rice, 1974). The congruence values 206 (Tucker, 1951) between the rotated loading matrix and the ideal loading matrix ranged from 0.88 207 to 0.96 . As the coefficients were all above the threshold of 0.85 , the factor similarity between the 208 rotated loading matrix and the ideal loading matrix was fair (Lorenzo-Seva \& Ten Berge, 2006). 209 Table 3 shows not only the loading values after rotation, but also the loadings of items on the 210 control scale (i.e., the acquiescence, AC). The procedure used to obtain a control scale was 211 previously proposed by Lorenzo-Seva \& Ferrando, 2009 Lorenzo-Seva \& Ferrando, 2009). The 212 method is based on the idea that in a scale where some items are worded in the opposite direction to 213 the other items, it is possible to identify acquiescent response style. In such a balanced scale, the 214 centroid helps to estimate the overall tendency of individuals to use systematically a particular value 
215 of the response scales independently of the worded direction of the items (i.e., to show an

216 acquiescent response style). In an initial step, the first centroid is computed, and it is taken as an

217 estimate of the loading values of items on an underlying acquiescent factor. If the scale is partially

218 balanced, a subset of balanced items is used to compute the first centroid, and then the unbalanced

219 set of items is projected on the first centroid. The variance explained by the first centroid is then

220 removed from the correlation matrix, and the residual correlation matrix is factor analyzed in order

221 to estimate the loading on the content factors. In addition, this first centroid can be understood as a

222 control scale: a scale that accounts for the variance due to the acquiescent response. Our results

223 show that some of the items properly loaded on the AC scale. This finding confirms the appropriate

224 use of a model in which AC response bias style was controlled. We were also able to validate that

225 the loadings of items on the content factors were free of AC using this model. Lastly, using the

226 loading values on the content factor, we demonstrated that the items in our adapted instrument were

227 well related with the corresponding expected scale

228

229

230 In addition, the inter-factor correlation values between content factors ranged between 0.22 and

231 0.32. While these inter-factor correlations are in general slightly lower than the original version of

232 the test by Mas-Herrero (0.22 to 0.46$)$, our results demonstrated that the scales were also correlated 233 in the French adaptation.

234

235 Finally, the reliability estimates computed on the basis of the factor scores of the scales were 0.93 , 236 0.96, 0.88, 0.91, and 0.93 for Musical Seeking, Emotion Evocation, Mood Regulation, Social 237 Reward and Sensory-Motor, respectively. None of the reliability estimates obtained in our 
238 analyses were below the threshold of 0.80 . In comparison, the corresponding reliability estimates

239 in the original pooled English and Spanish samples were 0.89, 0.88, 0.87, 0.78, and 0.93,

240 respectively (Mas-Herrero et al., 2013). Furthermore, the overall test (Music reward) in the

241 French translation showed an acceptable reliability (0.91), concordant with the reported value by

242 Mas-Herrero et al. (0.92). The distribution of the overall test scores (global sensitivity to music

243 reward) using the French translation was centered on a mean of 50, similar to that of the original

244 instrument (Figure 1). Likewise, the internal consistency for the overall French scale was 0.852 ,

245 with a 95\% confidence interval $[0.839,0.865]$. Globally, all our analyses demonstrated that the

246 fit obtained in the French translation was similar to that of the original English and Spanish

247 samples, indicating that the factorial structures are equivalent.

248

$249 \underline{\text { Discussion }}$

250

251 Our study described the translation and adaptation of the BMRQ into French and provided

252 analyses of the psychometric properties of the translated scale. Our results demonstrated that the

253 translated BMRQ has acceptable construct validity while keeping the factorial structure of the

254 original English and Spanish questionnaires. In general, the results that we obtained were similar

255 to those reported by the developers of the original instrument (Mas-Herrero et al., 2013). This

256 suggests that our translation procedure was successful.

257 The geographic distribution of French speakers encompasses over 30 countries throughout all

258 five continents (L'observatoire de la langue française, 2014). With such a diverse speaker

259 population, significant regional differences in the spoken language currently exist. In that

260 context, an internationally acceptable French adaptation of the BMRQ was required to 
261 accommodate researchers and clinicians across the French-speaking regions. This translated

262 BMRQ is born from a collaborative work between two bilingual groups in North America

263 (Montreal, Quebec) and Europe (Lyon, France). Efforts were made during the translation process

264 to remove all regional French influences. Each group first independently translated the original

265 English BMRQ into a locally acceptable French. Then, a consensus between the two translators

266 was obtained to produce the final international French version of the questionnaire. We believe

267 this collaboration was necessary to adapt the original BMRQ into a French that would be easily

268 understood by speakers around the French-speaking world. This belief is echoed in our results:

269 over 30 French-speaking countries are represented, and 5.5\% of participants learned French in

270 countries other than Canada (Quebec) or France. Finally, our association with the developers of

271 the original Spanish instrument (UL) ensured the French adaptation remained faithful to the

272 initial questionnaire.

274 While we collected responses from over 1000 participants, the majority were obtained from

275 France $(89.4 \%)$. This is partly a reflection of the differences in the number of French speakers

276 between the regions sampled: 6 million in Quebec compared to more than 77 million in the

277 European Union (L'observatoire de la langue française, 2014). In an effort to reduce sampling

278 bias effect, the music focus of the study was not explicitly stated in the test instructions when

279 administered to participants. This can be seen in the number of non-musicians among our

280 participants $(77.5 \%)$, a proportion that is similar to what has been reported in the original version

281 of the BMRQ. We therefore believe our sample is representative of the general French speaking

282 population and that sampling bias was not significant. 
284 Previous work by Ayotte (2002) and Peretz (2003) have established that approximately 4\% of

285 the population suffers from congenital amusia, a disorder of music processing that hinders their 286 ability to perceive, produce and enjoy music (Ayotte, Peretz \& Hyde, 2002; Peretz, Champod \& 287 Hyde, 2003). In contrast, some individuals suffer from general anhedonia, a deficit in 288 experiencing pleasure from widely different stimuli, usually in the context of depressive 289 disorders or neurodegenerative diseases such as Parkinson's (Loas et al., 1994; Assogna et al., 290 2011). Three case studies have also reported a form of acquired anhedonia specific to music that 291 resulted from strokes in limbic structures such as the amygdala, as well as areas of the temporo292 parietal cortex, inferior parietal cortex and insula (Mazzoni et al., 1993, Satoh et al., 2011, 293 Griffiths et al., 2004). In those neurologic and psychiatric patients, the use of a standardized tool 294 such as the BMRQ will help determine a loss in the capacity of feeling emotions through music. 295 However, the BMRQ can also be employed to explore music reward in healthy individuals. In 296 fact, a recent report by Mas-Herrero et al. (2014) was the first to identify a group of healthy 297 people for whom music is not rewarding (Mas-Herrero et al., 2014). The term coined - specific 298 musical anhedonia - refers to a unique subset of the population that draws no pleasure at all from 299 music despite being perfectly able to experience pleasure in other ways. Using a stepwise 300 regression analysis, Mas-Herrero et al. found the BMRQ score to be the only predictor of high301 pleasure or chill responses in all their participants (compared to other reward scales such as the 302 BIS/BAS). Their work has shown that the ability of music to induce pleasure may not be 303 universal, and that there may be individual differences in access to the reward system (Mas304 Herrero et al., 2014). To further understand the neural correlates behind musical pleasure and 
305 reward processing, further studies in that population are required and the BMRQ could prove to 306 be a very valuable tool.

307

308 Conclusion

309

310 The French version of the BMRQ appears valid and reliable. The addition of the French

311 adaptation to the previously available English and Spanish versions significantly increases the

312 reach of this scale. We believe it can not only serve as a valuable psychophysical tool in music

313 reward and emotion research, but its use could also be extended to emotion and reward research

314 in other domains and modalities, in which music can be used to test the specificity of a given

315 deficit. Clinical applications of the BMRQ include the examination of musical pleasure

316 experience in healthy individuals and in patients suffering from a wide variety of cognitive,

317 neurologic and auditory disorders.

318

319 The French BMRQ test is available online at the following URL:

320 www.brainvitge.org/bmrq_french.php

\section{Acknowledgements}

323 We thank Anne Caclin, Lesly Fornoni and Yohana Lévêque from the Lyon research team on

324 congenital amusia for their collaboration in this translation project into French as well as Gerard

325 Mick for help in distributing the announcement. We also thank Robert Zatorre for his scientific 
326

327

328

329

330

331

332

333

334

335

336

337

338

339

340

341

342

343

344

345

346

347

348

349

350

351

352

353

354

355

356

357

358

359

360

361

362

363

364

365

366

367

368

input and help in coordinating this effort.

\section{$\underline{\text { References }}$}

Assogna F, Cravello L, Caltagirone C, Spalletta G 2011. Anhedonia in Parkinson's disease: a systematic review of the literature. Movement disorders : official journal of the Movement Disorder Society 26:1825-1834.

Ayotte J, Peretz I, Hyde K 2002. Congenital amusia: A group study of adults afflicted with a music-specific disorder. Brain : a journal of neurology 125:238-251.

Blood AJ, Zatorre RJ 2001. Intensely Pleasurable Responses to Music Correlate with Activity in Brain Regions Implicated in Reward and Emotion. Proceedings of the National Academy of Sciences of the United States of America 98:11818-11823.

Browne, MW 1972. Oblique rotation to a partially specified target. British Journal of Mathematical and Statistical Psychology. 25:207-212.

Carver CS, White TL 1994. Behavioral Inhibition, Behavioral Activation, and Affective Responses to Impending Reward and Punishment: The BIS/BAS Scales. Journal of Personality and Social Psychology 67:319-333.

Chanda ML, Levitin DJ 2013. The neurochemistry of music. Trends in Cognitive Sciences 17:179-193.

Cross, I 2001. Music, cognition, culture, and evolution. Annals of the New York Academy of Sciences 930: 28-42.

Griffiths TD, Warren JD, Dean JL, and Howard D 2004. "When the feeling's gone": a selective loss of musical emotion. J Neurol Neurosurg Psychiatry 75: 344-345

Juslin PN, Västfjäll D 2008. Emotional responses to music: the need to consider underlying mechanisms. The Behavioral and brain sciences 31:559-75- discussion 575-621.

L'observatoire de la langue française. 2014. La langue française dans le monde. Available at http://www.francophonie.org/Rapports-Publications.html. Accessed on July 222015

Loas G, Salinas E, Pierson A, Guelfi JD, Samuel-Lajeunesse B 1994. Anhedonia and blunted affect in major depressive disorder. Comprehensive psychiatry 35:366-372.

Lorenzo-Seva U and Ferrando PJ 2009. Acquiescent responding in partially balanced multidimensional scales. British Journal of Mathematical and Statistical Psychology, 
371

372

373

374

375

376

377

378

379

380

381

382

383

384

385

386

387

388

389

390

391

392

393

394

395

396

397

398

399

400

401

402

403

404

405

406

407

408

409

410

$62: 319-326$

Lorenzo-Seva U and Rodriguez-Fornells, A 2006. Acquiescent responding in balanced multidimensional scales and exploratory factor analysis. Psychometrika, 71:769-777.

Mas-Herrero E, Marco-Pallares J, Lorenzo-Seva U, Zatorre RJ, Rodriguez-Fornells A 2013. Individual Differences in Music Reward Experiences. Music Perception: An Interdisciplinary Journal 31:118-138.

Mas-Herrero E, Zatorre RJ, Rodriguez-Fornells A, Marco-Pallares J 2014. Dissociation between Musical and Monetary Reward Responses in Specific Musical Anhedonia. Current Biology 24:699-704.

Mazzoni M, Moretti P, Pardossi L, Vista M, Muratorio A, Puglioli M 1993. A case of music imperception. J Neurol Neurosurg Psychiatry 56: 322.

Mellenbergh, GH 1994. A unidimensional latent trait model for continuous item responses. Multivariate Behavioral Research 28:175-184.

Menon V, Levitin DJ 2005. The rewards of music listening: Response and physiological connectivity of the mesolimbic system. NeuroImage 28:175-184.

Peretz I, Champod AS, Hyde K 2003. Varieties of musical disorders. The Montreal Battery of Evaluation of Amusia. Annals of the New York Academy of Sciences 999:58-75.

Satoh M, Nakase T, Nagata K, Tomimoto H 2011. Musical anhedonia: selective loss of emotional experience in listening to music. Neurocase 17: 410-417.

Ten Berge JMF and Kiers HAL 1991. A numerical approach to the approximate and the exact minimum rank of a covariance matrix. Psychometrika, 56:309-315.

Ten Berge JMF, Krijnen W, Wansbeek T, Shapiro A 1999. Some new results on correlationpreserving factor scores prediction methods. Linear Algebra and its Applications. 289:311318.

Torrubia R, Ávila C, Moltó J, Caseras X 2001. The Sensitivity to Punishment and Sensitivity to Reward Questionnaire (SPSRQ) as a measure of Gray's anxiety and impulsivity dimensions. Personality and Individual Differences 31:837-862.

Tucker, L. R. 1951. A method for synthesis of factor analysis studies (Personnel Research Section Report No. 984). Washington D.C.: Department of the Army. 
1

Global sensitivity to music reward scores using the French version of the Barcelona Music Reward Questionnaire

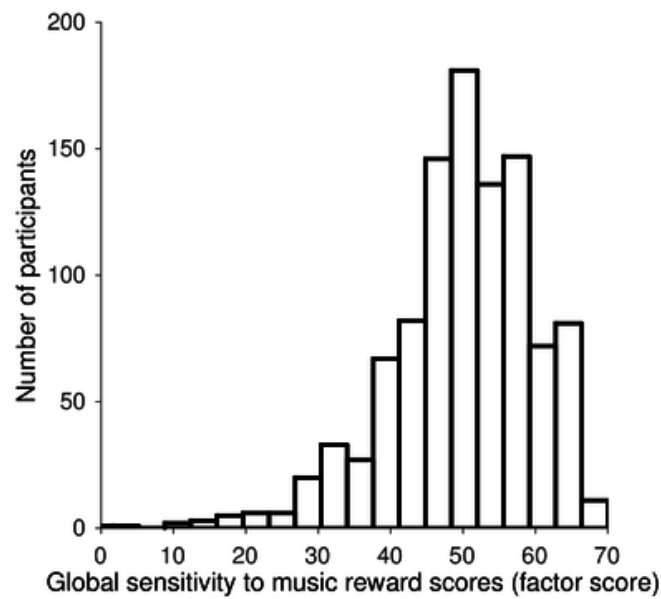


Table $\mathbf{1}$ (on next page)

Demographics 
1 Table 1. Demographics

2

\begin{tabular}{lc}
\hline \multicolumn{1}{c}{ Variable } & Participants \\
$(\mathbf{n}=\mathbf{1 0 2 7})$
\end{tabular}

3 


\section{Table 2 (on next page)}

Item by item comparison between the original Spanish scale and the adapted French version

(SD, standard deviation) 
1 Table 2. Item by item comparison between the original Spanish scale and the adapted French

2 version (SD, Standard deviation)

3

4

\begin{tabular}{lll}
\hline Item & $\begin{array}{c}\text { Original } \\
\text { Mean (SD) }\end{array}$ & $\begin{array}{c}\text { French } \\
\text { Mean }(\text { SD })\end{array}$ \\
\hline Q1 & $3.85(0.86)$ & $4.00(0.78)$ \\
Q2 & $1.70(0.92)$ & $1.77(1.01)$ \\
Q3 & $4.30(0.79)$ & $4.32(0.78)$ \\
Q4 & $4.17(0.91)$ & $4.32(0.88)$ \\
Q5 & $1.65(1.02)$ & $2.11(1.29)$ \\
Q6 & $3.74(0.86)$ & $3.63(0.99)$ \\
Q7 & $3.89(0.98)$ & $3.72(1.01)$ \\
Q8 & $4.53(0.70)$ & $4.49(0.76)$ \\
Q9 & $4.26(0.82)$ & $4.33(0.77)$ \\
Q10 & $3.96(1.03)$ & $3.61(1.24)$ \\
Q11 & $3.46(0.86)$ & $3.49(1.09)$ \\
Q12 & $3.55(1.06)$ & $3.50(1.36)$ \\
Q13 & $3.28(1.25)$ & $3.12(1.36)$ \\
Q14 & $4.35(0.78)$ & $4.29(0.85)$ \\
Q15 & $4.29(0.76)$ & $4.21(0.93)$ \\
Q16 & $3.82(0.92)$ & $3.69(0.99)$ \\
Q17 & $2.29(1.12)$ & $2.17(1.13)$ \\
Q18 & $3.94(0.88)$ & $4.08(1.01)$ \\
Q19 & $4.11(0.98)$ & $4.12(0.84)$ \\
Q20 & $4.00(0.91)$ & $4.21(0.90)$ \\
& &
\end{tabular}

5

6

7 


\section{Table 3(on next page)}

Factorial loading matrix for each item of the adapted French version of the questionnaire.

Salient loading values (i.e., values larger than absolute 0.4 ) in the content factors are printed in bold face. 
1 Table 3. Factorial loading matrix for each item of the adapted French version of the

2 questionnaire. Salient loading values (i.e., values larger than absolute 0.4 ) in the content factors 3 are printed in bold face.

4

\begin{tabular}{ccccccc}
\hline Item & Acquiescence & $\begin{array}{c}\text { Music } \\
\text { seeking }\end{array}$ & $\begin{array}{c}\text { Emotion } \\
\text { evocation }\end{array}$ & $\begin{array}{c}\text { Mood } \\
\text { regulation }\end{array}$ & $\begin{array}{c}\text { Sensori- } \\
\text { motor }\end{array}$ & Social \\
\hline Q11 & 0.047 & $\mathbf{0 . 7 9 0}$ & -0.060 & 0.108 & 0.108 & -0.056 \\
Q2 & 0.473 & $\mathbf{- 0 . 6 2 9}$ & 0.019 & -0.290 & 0.005 & -0.037 \\
Q7 & 0.557 & $\mathbf{0 . 6 2 5}$ & 0.005 & 0.087 & -0.047 & 0.099 \\
Q17 & 0.193 & $\mathbf{0 . 6 0 5}$ & 0.126 & -0.173 & -0.077 & 0.270 \\
Q12 & -0.039 & 0.004 & $\mathbf{0 . 9 0 4}$ & -0.122 & 0.083 & -0.080 \\
Q8 & 0.057 & 0.056 & $\mathbf{0 . 8 5 6}$ & 0.021 & -0.002 & -0.001 \\
Q18 & 0.098 & 0.031 & $\mathbf{0 . 6 8 6}$ & 0.093 & -0.057 & 0.100 \\
Q3 & -0.055 & -0.117 & $\mathbf{0 . 6 3 4}$ & 0.208 & -0.041 & 0.059 \\
Q14 & -0.052 & 0.034 & -0.042 & $\mathbf{0 . 7 4 8}$ & 0.066 & 0.134 \\
Q9 & 0.007 & 0.218 & 0.101 & $\mathbf{0 . 6 8 0}$ & 0.057 & -0.134 \\
Q4 & -0.185 & 0.056 & 0.072 & $\mathbf{0 . 6 6 5}$ & 0.032 & -0.022 \\
Q19 & -0.023 & 0.131 & 0.241 & $\mathbf{0 . 6 4 1}$ & 0.046 & -0.011 \\
Q10 & 0.050 & 0.059 & -0.020 & -0.050 & $\mathbf{0 . 9 7 5}$ & -0.106 \\
Q5 & 0.236 & -0.055 & -0.005 & 0.253 & $\mathbf{- 0 . 9 3 3}$ & 0.044 \\
Q20 & -0.013 & -0.041 & 0.013 & 0.314 & $\mathbf{0 . 5 2 7}$ & 0.158 \\
Q15 & -0.005 & -0.247 & 0.002 & 0.363 & $\mathbf{0 . 4 4 3}$ & 0.311 \\
Q1 & 0.018 & 0.033 & 0.039 & -0.044 & -0.052 & $\mathbf{0 . 7 0 5}$ \\
Q6 & 0.085 & 0.225 & -0.121 & 0.014 & 0.020 & $\mathbf{0 . 7 0 4}$ \\
Q13 & 0.082 & -0.039 & 0.114 & -0.013 & 0.144 & $\mathbf{0 . 5 9 1}$ \\
Q16 & 0.091 & 0.124 & 0.138 & -0.115 & 0.150 & $\mathbf{0 . 5 2 6}$ \\
\hline
\end{tabular}

